\title{
Phylogenetic Diversity Theory Sheds Light on the Structure of Microbial Communities - Supporting Information
}

\author{
James P. O’Dwyer, Steven W. Kembel, Jessica L. Green
}

\section{Figure S1}

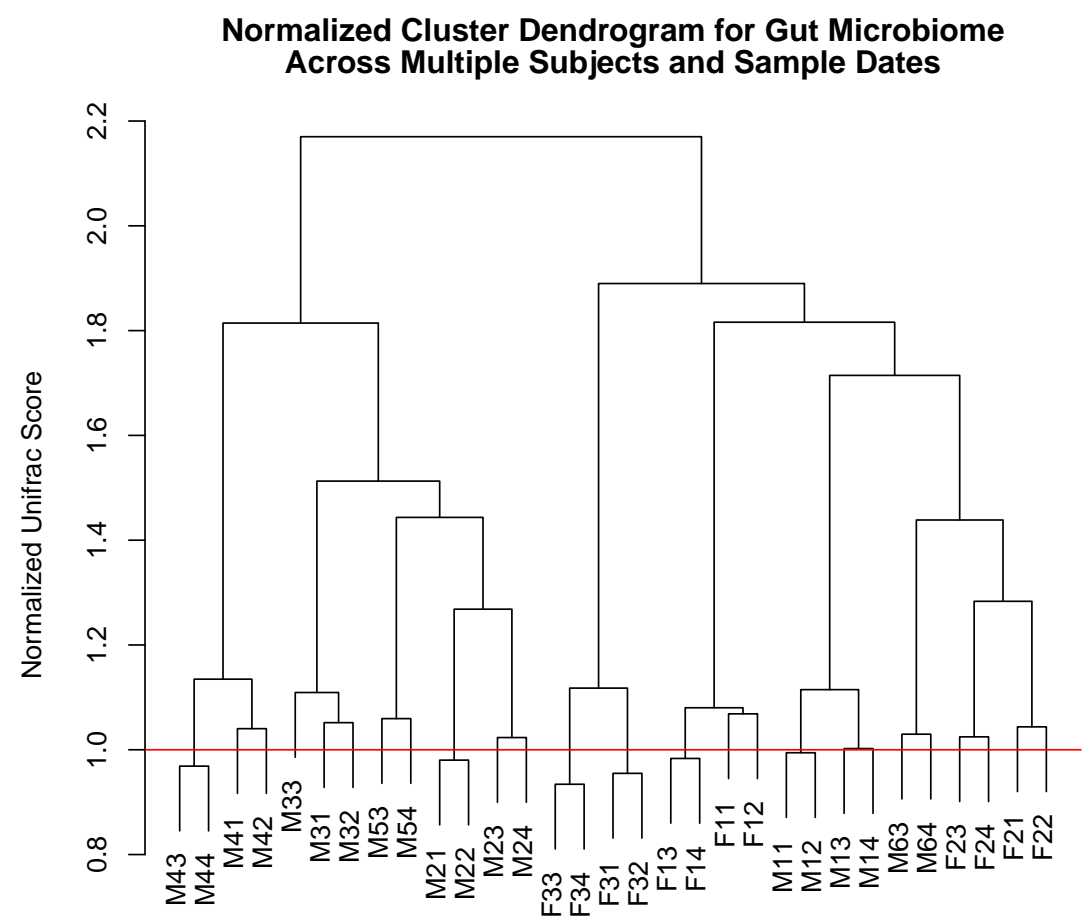

Figure S1 Hierarchical clustering for gut samples. Distances are defined using the Unifrac metric, but normalized by expectation values corresponding to appropriately-sized random samples from the gut metacommunity. Each sample is labeled by a letter ( $\mathrm{M}$ or $\mathrm{F}$ indicating gender of the subject), and two numbers: the first number indicates subject, the second sample date. Sample dates 1 and 2 are on consecutive days, as are 3 and 4 . The red line corresponds to a distance between a pair of samples which is equal to the unifrac score for two random samples, and so we see that a number of pairs of samples taken from the same subject on consecutive days are more similar than random samples. No other pairs are more similar than random. 